\title{
Tumor Budding Versus Mismatch Repair Status in Colorectal Cancer - An Exploratory Analysis
}

\author{
MIA KARLBERG ${ }^{1,2}$, KRISTINA STENSTEDT $^{2,3}$, MARJA HALLSTRÖM $^{1}$, \\ PETER RAGNHAMMAR $^{1}$, CLAES LENANDER $^{1}$ and DAVID EDLER ${ }^{2,3}$ \\ ${ }^{1}$ Department of Oncology-Pathology, Karolinska Institute, Stockholm, Sweden; \\ ${ }^{2}$ Center of Digestive Diseases, Karolinska University Hospital, Stockholm, Sweden; \\ ${ }^{3}$ Department of Molecular Medicine and Surgery, Karolinska Institute, Stockholm, Sweden
}

\begin{abstract}
Background: Tumor budding and a proficient mismatch repair (pMMR) status are considered adverse prognostic factors in colorectal cancer (CRC). The aim of this pilot study was to assess tumor budding in primary CRC with pMMR versus that with deficient mismatch repair (dMMR). Materials and Methods: Tumor budding was retrospectively examined in the tumor from 134 patients with stage II and stage III CRC with known MMR status. The 29 available dMMR cases who developed recurrence or distant metastases (met+) were matched with a dMMR group with no recurrence or metastases (met-), and the pMMR/met+ group with pMMR/met-cases. Results: Using tumor budding cut-offs of 5 and 10, a significantly higher percentage of high-grade tumor budding $(\geq 5$ and $\geq 10)$ was only found in the $d M M R / m e t+$ compared to $p M M R / m e t+$ subgroup $(p=0.01$ and $p=0.02$, respectively). Conclusion: A significantly higher grade of tumor budding was observed in the dMMR/met+ group, suggesting that tumor budding can provide prognostic information for patients with a dMMR status.
\end{abstract}

Colorectal cancer (CRC) is the third most common cancer as well as the third leading cause of cancer-related death in the Western world (1). The TNM staging system as well as established tumor-related prognostic factors are used clinically for assessment regarding risk for recurrence and in the decision to recommend adjuvant chemotherapy in patients with stage II and stage III CRC (2-4).

This article is freely accessible online.

Correspondence to: Mia Karlberg MD, Department of OncologyPathology, Karolinska Institute and Center of Digestive Diseases, Karolinska University Hospital, S-171 76 Stockholm, Sweden. Tel: +46 851779680, Fax: +468339031, e-mail: mia.karlberg@sll.se

Key Words: Tumor budding, mismatch repair, colorectal cancer.
Research in CRC continues to focus on finding prognostic and predictive markers that will help optimize treatment and thus improve overall survival. A major challenge is to identify patients at risk of developing future distant metastases after their initial diagnosis and curative surgery. This is particularly relevant for patients with stage II CRC of which the majority are cured by surgical resection but approximately $20-25 \%$ will go on to develop metastases within 5 years $(5,6)$. The mismatch repair (MMR) status is considered a prognostic factor in early sporadic CRC, in which a deficient MMR status (dMMR) or high-level microsatellite instability is associated with a reduced rate of metastatic disease despite lymph node positivity $(4,7)$. However, dMMR status seems in the adjuvant setting predictive of a poorer response to 5-fluorouracil-based chemotherapy $(8,9)$.

Studies in CRC have shown that tumor budding has prognostic potential, where higher grades of tumor budding are associated with a higher recurrence rate and a worse overall survival regardless of the primary pathological stage (10-12). Tumor budding, specifically peri-tumoral budding, is described as the transition from glandular structures to detached, dedifferentiated single cancer cells or clusters with fewer than five cancer cells at the invasive margin of the tumor $(13,14)$. The classification of tumor budding into low-grade or high-grade is suggested to reflect cell de-differentiation in which higher grades correlate with the development of regional and distant metastases and a poorer prognosis $(12,13,15)$. The process of de-differentiation seen in tumor budding is thought to be an example of epithelial-mesenchymal transition and activation of the wingless gene (Wnt) signaling pathway, as seen in in vitro studies $(13,16,17)$. Tumors with proficient MMR (pMMR) or which are microsatellite-stable are considered chromosomally instable, typically having mutations in the adenomatous polyposis coli gene (APC), and are mainly driven by activation of the Wnt pathway $(16,18)$. A higher grade of budding has been observed in pMMR or 
microsatellite-stable tumors compared to tumors with dMMR or high-level microsatellite instability $(13,16,19)$.

Tumor budding is a prognostic marker in primary CRC that may aid in stratification of recurrence risk, especially in stage II (T1-T3N0), and thus influence treatment decisions regarding recommendation of adjuvant chemotherapy to the patient $(20,21)$.

To avoid underestimation of tumor budding, the neoplastic epithelium can be highlighted by pan-cytokeratin immunostaining (22-25). At the time this study was done there was no established method or consensus for assessment of or cut-off values for tumor budding $(24,26,27)$. The methods used to date in assessing tumor budding are categorized as either having a qualitative or scoring approach versus a quantitative or counting approach $(12,28)$. A quantitative assessment of tumor budding and two cut-off levels were chosen for this exploratory study.

The aim was to investigate primary CRC tumors with pMMR compared with $\mathrm{dMMR}$ and assess whether the grade of tumor budding differed according to whether local recurrence or metastases developed or not.

\section{Materials and Methods}

The patient material was derived from a previously described group of 1,006 patients with stage II and III CRC from 59 hospitals in Sweden who underwent surgery between 1991-1997. All patients were included in the adjuvant Nordic trial where a total of 2,224 patients were randomized to either surgery alone or surgery followed by 5-fluorouracil-based adjuvant chemotherapy (29).

The MMR status was previously analyzed as a prognostic marker in a study of these 1,006 Swedish patients out of the total 2,224 patients in the Nordic trials (30) (Figure 1). The parameters of clinical outcome were obtained from the Regional Cancer Center in Sweden. Censoring of data occurred at 120 months after surgery.

From the group of 1,006 patients analyzed for MMR status, 35 patients with tumors classified with dMMR that had developed recurrence or distant metastases (dMMR/met+) and 29 tumor samples out of these 35 were available for further analysis of tumor budding. This group of 29 was then matched according to age, gender and treatment (surgery alone vs. surgery with adjuvant chemotherapy) to 33 patients with dMMR tumor status that had no recurrence or metastases (dMMR/met-), 42 patients with pMMR tumor and no recurrence or metastases (pMMR/met-) and 30 patients with pMMR tumor and recurrence or distant metastases (pMMR/met+) (Figure 1). The assignment of a total of 134 patients into these four categories was carried out in order to analyze differences in tumor budding between the categories.

Sixteen patients with rectal cancer, $12 \%$ of the total study population, with known MMR/recurrence or metastasis status, who did not receive preoperative radiotherapy were also included and were evenly distributed between the four groups.

Laboratory methods. Two formalin-fixed paraffin-embedded samples, each $4 \mu \mathrm{m}$-thick, were taken from different areas of each primary colorectal tumor. The MMR status was assessed in our earlier study using immunohistochemistry with mouse immunoglobulin G monoclonal antibodies. Clone G168-15 (BD PharMingen, San Diego, CA, USA) at 1:100 dilution was used for analyzing the mutL homolog1 (MSH) gene protein product and clone FE11 (Oncogene Research Products, Boston, MA, USA) at 1:100 dilution was used in the mutS homolog 2 (MSH2) gene protein product analysis. The laboratory method for the analysis of MMR status has been described in detail elsewhere (30).

To aid in the detection of tumor budding, immunohistochemicaI analysis was performed using staining with monoclonal mouse antibody pan-cytokeratin MNF-116 (1:75; DakoCytomation, Glostrup, Denmark). The system used was horseradish peroxidase with EnVision ${ }^{\mathrm{TM}}$ (DakoCytomation).

Scoring. Assessment of tumor budding with MNF-116 staining was performed by two independent investigators (MK and CL) blinded to both clinical and pathological data. Tumor budding was defined as isolated single de-differentiated cancer cells or clusters of fewer than five such cells at the invasive front (31). One of the two available tumor samples per case was chosen as being most suitable for tumor budding investigation after scanning the invasive front with a multiheaded microscope at low power magnification $(10 \times)$. Magnification of $20 \times$ was used for counting the number of tumor buds along the entire invasive margin of each tumor section in a specified area defined by a grid (area of the grid $0.05 \mathrm{~mm}^{2}$ ). Two different cutoffs were used: the median tumor budding value of 5 from this study and the cut-off of 10 which has been used in other studies as an optimal cut-off with hemotoxylin and eosin (H\&E) staining $(31,32)$ or with cytokeratin staining (33). Using these two cut-off values, the degree of budding was categorized into two groups, low-grade (below the cut-off) and high-grade (at or above the cut-off). Examples of lowand high-grade budding are shown in Figure 2.

Statistical analysis. Chi-square tests were used to compare the differences in distributions of patient characteristics and tumor budding grades between the groups. Differences with values of $p<0.05$ were considered statistically significant. Linear correlation between the two observers' tumor budding counts was assessed using Pearson correlation coefficient $(r)$. The intra-class correlation coefficients (ICC) assessed interobserver agreement, where values range from 0 (no agreement) to 1 (perfect agreement). The Cox univariate regression analysis was used to determine possible correlation of MMR status and grade of tumor budding with disease-free (DFS) and overall survival (OS). DFS was defined as time from surgery to the first event of local recurrence, presence of distant metastases or death of any reason and OS was defined as time from surgery to death.

Log-rank test was used to detect differences in time to recurrence between the dMMR/met+ and pMMR/met+ groups. Cox proportional hazards regression models were used for multivariate analysis and the Kaplan-Meier method for estimation of survival curves. All statistical tests were performed using Statistica version 10, StatSoft Scandinavia AB, Uppsala, Sweden.

\section{Results}

Patients. The patient and tumor characteristics are listed in Table I. Groups were matched according to age, gender and treatment arm.

The 5-year survival rate for the entire study population of 134 patients was 52\%, with a median OS of 60 months and a median DFS of 49 months. For the entire group there was no 


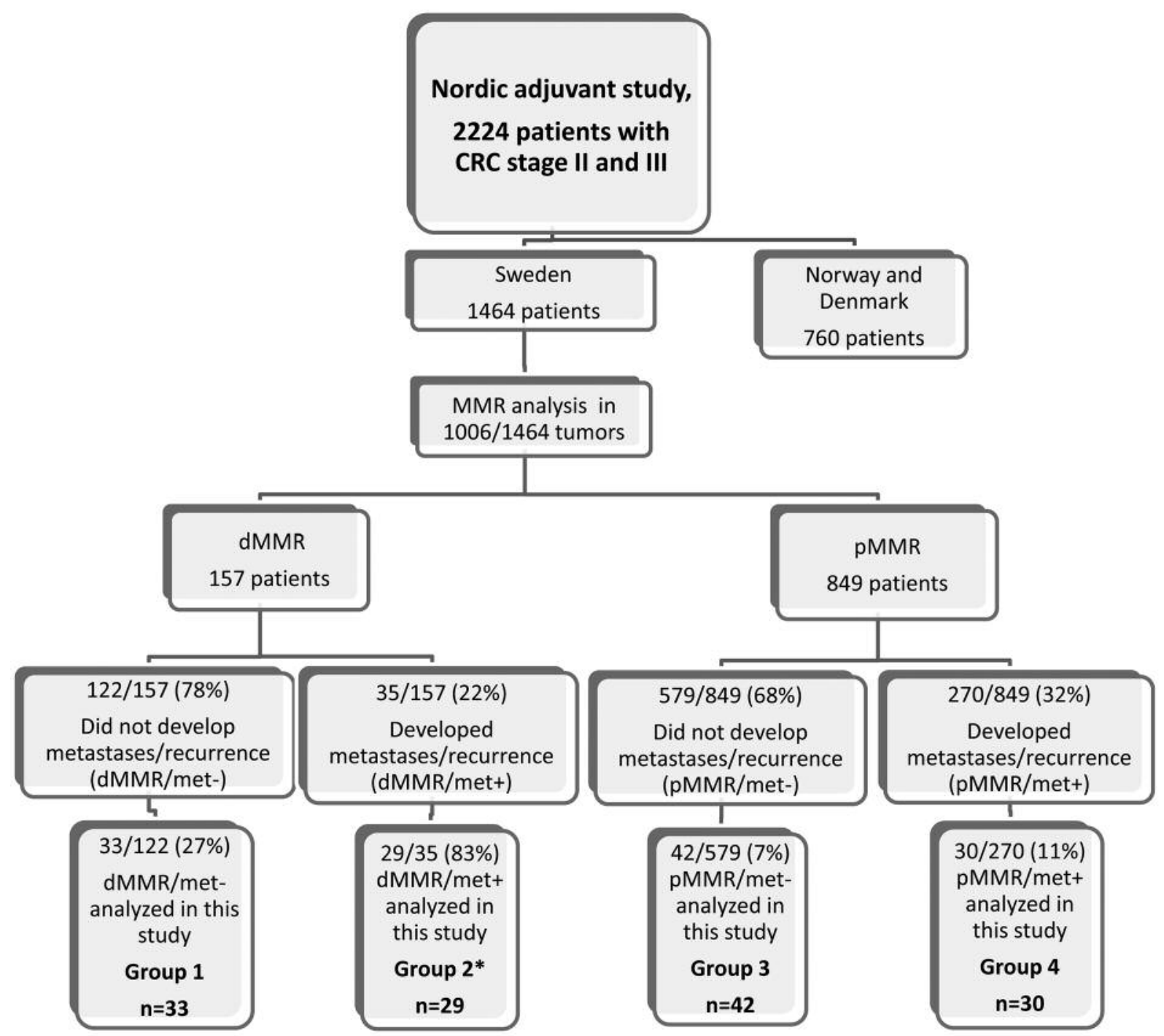

Figure 1. Flow chart of patients included in our study. MMR, Mismatch repair; dMMR, deficient MMR; pMMR, proficient MMR; met-, no recurrence or distant metastases; met+, with recurrence or distant metastases; Group $2 *$ : Index group identified and then matched to groups 1,3 and 4 according to age, gender and treatment.

significant difference in OS between patients with stage II and and those with stage III CRC. Survival data were censored at 120 months after surgery. Median follow-up of surviving patients was 100 months (range $=62-120$ months). Overall survival for the different groups is shown in Figure 3.

MMR status. For the tumors located proximal to the left colic or splenic flexure, $40(56 \%)$ were dMMR and 31 (44\%) were pMMR, whereas of tumors distal of the colic flexure site, 22 $(35 \%)$ were dMMR and $41(65 \%)$ were pMMR $(p=0.05)$. Analysis showed that in the Met+ group, the number of patients who developed metastases or local recurrence in the first year was $52 \%(15 / 29)$ in the dMMR group, while it was only $13 \%(4 / 30)$ in the pMMR group. However, the difference in time to recurrence was not significant $(p=0.4)$.

Tumor budding. The mean tumor budding count was 7 ( $\mathrm{SD} \pm 6$ ), the median was 5 , and the range was $0-35$. In the dMMR subgroup, the mean tumor budding count was 8 $(\mathrm{SD} \pm 7.3)$, the median 5.5, and the range was 0.26-31.3, while in the pMMR group, the mean was $6.4(\mathrm{SD} \pm 5.17)$, the median 5 and the range was 0.69 to 34.9 .

There was substantial agreement between the investigators on the budding count $(p<0.001, \mathrm{r}=1.0 ; \mathrm{ICC}=0.99)$. 
Table I. Patients and tumor characteristics stratified by study group.

\begin{tabular}{|c|c|c|c|c|c|c|}
\hline Characteristic & Total $(n=134)$ & dMMR/met- $(\mathrm{n}=33)$ & $\mathrm{dMMR} /$ met+ $(\mathrm{n}=29)$ & pMMR/met- $(\mathrm{n}=42)$ & $\mathrm{pMMR} / \mathrm{met}+(\mathrm{n}=30)$ & $p$-Value \\
\hline \multicolumn{7}{|l|}{ Age (years) } \\
\hline$\leq 66$ & $67(50 \%)$ & $16(48 \%)$ & $19(66 \%)$ & $19(45 \%)$ & $13(43 \%)$ & \multirow[t]{2}{*}{ NS } \\
\hline$>66$ & $67(50 \%)$ & $17(52 \%)$ & $10(34 \%)$ & $23(55 \%)$ & $17(57 \%)$ & \\
\hline \multicolumn{7}{|l|}{ Gender } \\
\hline Male & $69(51 \%)$ & $14(42 \%)$ & $17(59 \%)$ & $23(55 \%)$ & $15(50 \%)$ & \multirow[t]{2}{*}{ NS } \\
\hline Female & $65(49 \%)$ & $19(58 \%)$ & $12(41 \%)$ & $19(45 \%)$ & $15(50 \%)$ & \\
\hline \multicolumn{7}{|l|}{ Tumor site } \\
\hline Proximal colon* & $71(53 \%)$ & $25(76 \%)$ & $15(52 \%)$ & $19(45 \%)$ & $12(40 \%)$ & \multirow[t]{2}{*}{0.02} \\
\hline Distal colon + rectum & $63(47 \%)$ & $8(24 \%)$ & $14(48 \%)$ & $23(55 \%)$ & $18(60 \%)$ & \\
\hline \multicolumn{7}{|l|}{ Stage } \\
\hline II & $43(32 \%)$ & $5(15 \%)$ & $8(28 \%)$ & $17(40 \%)$ & $13(43 \%)$ & \multirow[t]{2}{*}{0.05} \\
\hline III & $91(68 \%)$ & $28(85 \%)$ & $21(72 \%)$ & $25(60 \%)$ & $17(57 \%)$ & \\
\hline \multicolumn{7}{|l|}{ No. of analyzed LN } \\
\hline $0-11$ & $84(63 \%)$ & $16(49 \%)$ & $19(66 \%)$ & $25(59 \%)$ & $24(80 \%)$ & \multirow[t]{3}{*}{0.06} \\
\hline$\geq 12$ & $21(16 \%)$ & $7(21 \%)$ & $1(3 \%)$ & $10(24 \%)$ & $3(10 \%)$ & \\
\hline Missing data & $29(21 \%)$ & $10(30 \%)$ & $9(31 \%)$ & $7(17 \%)$ & $3(10 \%)$ & \\
\hline \multicolumn{7}{|l|}{ Grade of differentiation } \\
\hline Poor & $36(27 \%)$ & $13(39 \%)$ & $10(35 \%)$ & $8(19 \%)$ & $5(17 \%)$ & \multirow[t]{4}{*}{ NS } \\
\hline Moderate & $90(67 \%)$ & $20(61 \%)$ & $16(55 \%)$ & $32(76 \%)$ & $22(73 \%)$ & \\
\hline Well & $6(4 \%)$ & $0(0 \%)$ & $2(7 \%)$ & $2(5 \%)$ & $2(7 \%)$ & \\
\hline Missing data & $2(2 \%)$ & & $1(3 \%)$ & & $1(3 \%)$ & \\
\hline \multicolumn{7}{|l|}{ Adjuvant chemotherapy } \\
\hline Surgery & $62(46 \%)$ & $15(45 \%)$ & $14(48 \%)$ & $20(48 \%)$ & $13(43 \%)$ & \multirow[t]{2}{*}{ NS } \\
\hline Surgery + adjuvant CT & $72(54 \%)$ & $18(55 \%)$ & $15(52 \%)$ & $22(52 \%)$ & $17(57 \%)$ & \\
\hline
\end{tabular}

dMMR, Deficient mismatch repair; pMMR, proficient mismatch repair; met-, no recurrence or distant metastases; met+, with recurrence or distant metastases; LN, lymph node; *, to splenic flexure; CT, chemotherapy.

The cut-off of 5 tumor buds (median) and the cut-off of 10 tumor buds were used to analyze the distribution of tumor budding in the four different groups (Table II). Budding was similarly distributed in the group of patients treated with surgery alone compared with the group treated with surgery and adjuvant chemotherapy.

Tumor budding and MMR status. Cut-off of 5: In the $\mathrm{dMMR} /$ met+ group, the frequency of high-grade budding was significantly higher (72\%) compared to the dMMR/metgroup (39\%) ( $p=0.009)$. A significantly higher frequency of high-grade tumor budding was also seen in dMMR/met+ (72\%) compared to pMMR/met+ $(40 \%)(p=0.01)$. No other significant differences using this cut-off were seen when comparing all the other groups.

Cut-off of 10: With the higher cut-off, $25 \%$ in the entire study group were classified as high-grade tumor budding compared to $52 \%$ when using the tumor budding cut-off of 5 as shown in Table II. High-grade tumor budding was significantly more frequent in the dMMR/met+ group (45\%) compared with the $\mathrm{dMMR} /$ met- group (21\%) ( $p=0.047)$. Using this higher cut-off, the dMMR/met+ group had a significantly higher frequency of high-grade tumor budding (45\%) compared to the pMMR/met$(19 \% ; p=0.012)$ and $\mathrm{pMMR} / \mathrm{met}+(17 \% ; p=0.02)$ groups.
In our study, no significant survival advantages were seen for patients according to tumor budding, regardless of the cut-off used.

\section{Discussion}

This study was designed to evaluate tumor budding in primary colorectal tumors with regards to MMR status as well as local recurrence/metastases. When comparing the four groups using two different cut-offs, we found a significantly higher frequency of high-grade tumor budding in the $\mathrm{dMMR} / \mathrm{met}+$ group. No significant survival advantage for the group with low-grade tumor budding was found in our study, regardless of the cut-off used. However, reliable differences in survival are difficult to establish due to the small number of patients in the study and the prearranged subgroups.

To our knowledge no other study has examined tumor budding looking specifically at subgroups divided according to MMR status as well as recurrence/metastases.

Material for the study was derived from 1,006 patients, of which there were only 35 with dMMR and who developed metastases/local recurrence. This limitation is probably due to the inherent biology of dMMR CRC which has a better prognosis and thus tend not to recur or 
A

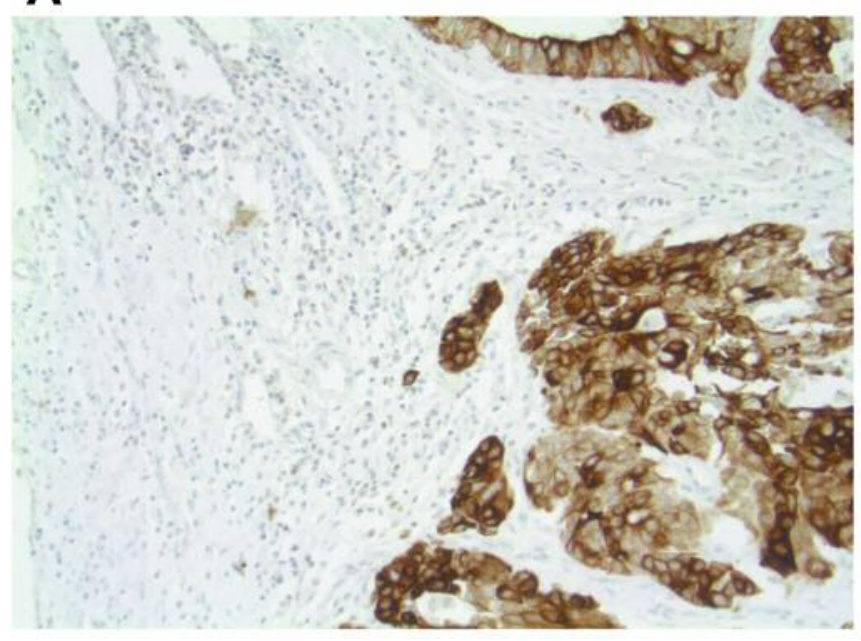

B

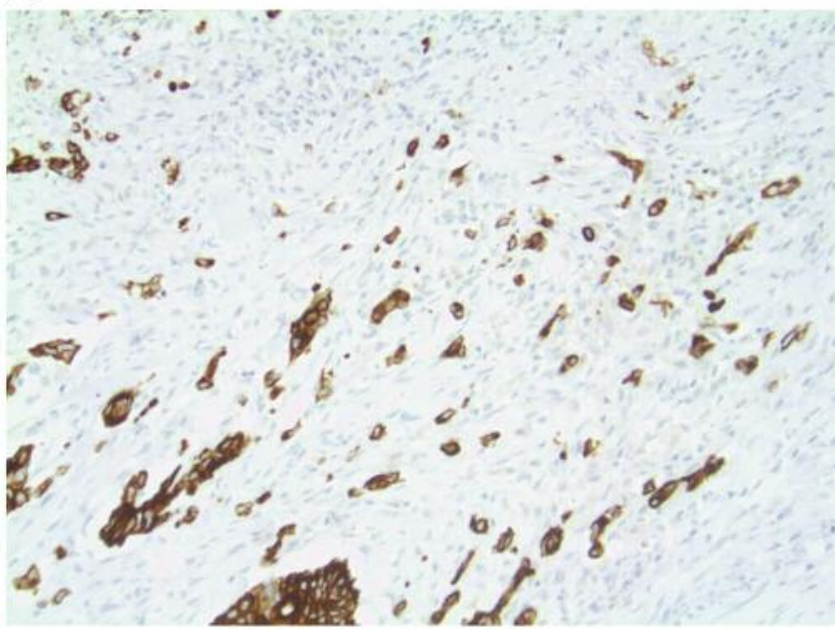

Figure 2. A: Low-grade tumor budding, 20x; B: high-grade tumor budding, 20x.

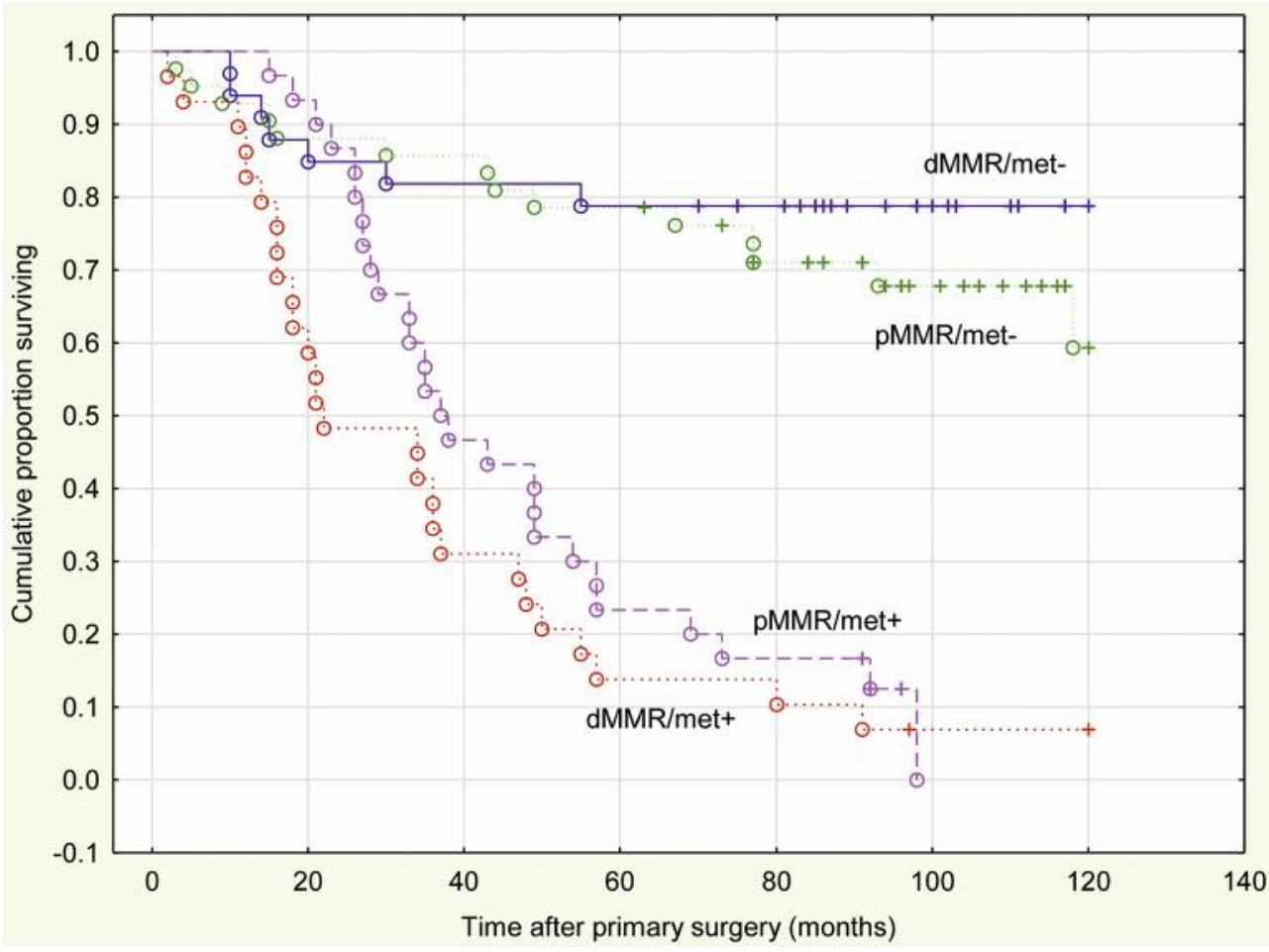

Figure 3. Overall survival in the four different groups. dMMR, Deficient mismatch repair (MMR); pMMR, proficient MMR; met-, no recurrence or distant metastases; met+, with recurrence or distant metastases.

metastasize. The inherent biology of dMMR tumors most likely also accounts for the significant difference in the distribution of tumor site where more tumors in the $\mathrm{dMMR} /$ met- group were located in the proximal colon. The low number of lymph nodes removed or analyzed in this study is due to the less accurate staging process during the time period 1991-1997 in which the Nordic adjuvant trials took place (29). 
Table II. Distribution of tumor budding in study groups.

\begin{tabular}{lccccrr}
\hline Cut-off & Class & Total $(\mathrm{n}=134)$ & dMMR/met- $(\mathrm{n}=33)$ & dMMR/met+ $(\mathrm{n}=29)$ & pMMR/met- $(\mathrm{n}=42)$ & pMMR/met+ (n=30) \\
\hline 5 & Low $(<5)$ & $64(48 \%)$ & $20(61 \%)$ & $8(28 \%)$ & $18(43 \%)$ & $18(60 \%)$ \\
& High $(\geq 5)$ & $70(52 \%)$ & $13(39 \%)$ & $21(72 \%)$ & $24(57 \%)$ & $12(40 \%)$ \\
10 & Low $(<10)$ & $101(75 \%)$ & $26(79 \%)$ & $16(55 \%)$ & $34(81 \%)$ & $25(83 \%)$ \\
& High $(\geq 10)$ & $33(25 \%)$ & $7(21 \%)$ & $13(45 \%)$ & $8(19 \%)$ & $5(17 \%)$ \\
\hline
\end{tabular}

dMMR, Deficient mismatch repair; pMMR, proficient mismatch repair; met-, no recurrence or distant metastases; met+, with recurrence or distant metastases.

As described in our earlier study (30), staining for MMR proteins was carried out for MSH2 and MLH1 and in cases where MLH1 staining was difficult to interpret even the protein product of the MMR gene postmeiotic segregation increased (Saccharomyces cerevisiae) 2 (PMS2) was stained. The majority of dMMR cases are found when staining the two proteins (MSH2 and MLH1), with a reported sensitivity of approximately $92 \%$ and a specificity of $100 \%$ (34).

When looking at MMR status with no regard to recurrence/metastases, compared to other similar studies, this study found a higher percentage of cases with high-grade tumor budding in the dMMR group and not in the pMMR group (Table III) $(19,35,36)$. These other studies used H\&E-stained slides except for Lugli et al. (19), as well as different assessment methods for tumor budding and cut-offs. As mentioned previously the design of the present study could be a possible explanation for this difference.

The higher tendency of tumor budding in pMMR tumors is thought to be driven by mutations in the $A P C$ gene and thereafter activation of the Wnt pathway $(15,18)$. Although dMMR tumors are not associated with tumor budding, in our study, a significant higher frequency of high-grade tumor budding was observed in the dMMR group in which patients developed metastases compared to the other groups. One study by Shinto et al. found that dMMR tumors had lower $\beta$-catenin expression in tumor buds compared to pMMR tumors, suggesting that Wnt signaling is not the only mechanism that leads to tumor budding (37). They also proposed that although dMMR (MLH1-loss) CRCs with high-grade tumor budding often have less local invasiveness, there may be an increased likelihood for vessel involvement and nodal metastases due to the loss of cell cohesion and thus an increased level of de-differentiation (37).

In one study by Lugli et al., intratumoral budding in CRC, analyzed by using immunostain for cytokeratin 22, was strongly related to peritumoral budding and was associated with a poorer outcome for those with high intratumoral budding independently of MMR status (19). Another recent study by van Wyk et al. analyzed tumor budding in 297 specimens from patients operated for primary stage I-III CRC, and assessed the presence of budding in H\&E slides
Table III. Clinicopathological studies analyzing high-grade vs lowgrade tumor budding with regards to mismatch repair (MMR) status.

\begin{tabular}{lccc}
\hline Author (Ref) & Cut-off & pMMR & dMMR \\
\hline Lugli et al. (16) & $\leq 6$ : Low PTB & $21 \%$ & $34 \%$ \\
& $>6$ : High PTB & $79 \%$ & $66 \%$ \\
& $\leq 6$ : Low ITB & $56 \%$ & $64 \%$ \\
& $>6$ : High ITB & $44 \%$ & $36 \%$ \\
Kevans et al. (30) & Low PTB* & $52 \%$ & $74 \%$ \\
& High PTB** & $48 \%$ & $26 \%$ \\
Karlberg et al. (this study) & $<5:$ Low PTB & $50 \%$ & $45 \%$ \\
& $\geq 5$ : High PTB & $50 \%$ & $55 \%$ \\
& $<10:$ Low PTB & $82 \%$ & $68 \%$ \\
& $\geq 10:$ High PTB & $18 \%$ & $32 \%$ \\
Wyk et al. (31) & $<20:$ Low PTB & $15 \%$ & $85 \%$ \\
& $\geq 20:$ High PTB & $85 \%$ & $15 \%$ \\
\hline
\end{tabular}

pMMR, proficient MMR; dMMR, deficient MMR; PTB, peritumoral budding; ITB, intratumoral budding; SD, standard deviation; ND, no data given; *low PTB (median=0); **high PTB (median $\geq 1$ ).

(36) using a 10 high-powered field method described in Karamitopoulou et al. (33). They used a cut-off of 20 for high budding based on a receiver operating characteristic curve analysis and found that in patients with known MMR status $(n=215)$, high budding was associated with a significantly lower 5-year OS for both pMMR and dMMR, although the number of patients in the dMMR group was low (36) (Table III).

The difficulty in implementing assessment of tumor budding in daily diagnostic practice is largely due to the wide heterogeneity in assessment methods and cut-off values and thus the lack of consensus on which is the best method $(26,27,38)$. A review by Zlobec (38) recommends scoring of tumor budding according to the method established by Karamitopolou et al. using pan-cytokeratin staining and the average of tumor budding in 10 high-power fields (HPFs) with the cut-off of 10 which was determined by receiver operating characteristic curve analysis with OS as end-point (33). In our study, we explored tumor budding variation using two cut-offs based on the median tumor budding value 
of 5 and a higher cut-off of 10 which was also used in the study of Karamitopoulou et al. (33) as well as other studies using H\&E-stained sections $(31,32)$. In the study of Kevans et al. (Table III) the median tumor budding value was also used to separate the low-grade from the high-grade tumor budding group (35). Another study, Park et al. stratified tumor budding based on the quartiles of the distribution of intensity. In their study, which did not incorporate MMR status, the best indicator for separating patients with regard to survival was the third-quartile group (intensity of 9). They found a significant less favorable 5-year DFS and OS outcomes for the group of patients with a budding intensity higher than 9 (39).

To account for the heterogeneity of budding, we chose to analyze the entire invasive front rather than hot-spot areas as was done in other studies $(40,41)$. Our study showed a mean tumor budding of $6.44(\mathrm{SD} \pm 5.17)$ for the pMMR group and $8(\mathrm{SD} \pm 7.3)$ for the dMMR group compared to another study (CRC stage II-III, $\mathrm{n}=297)$ showing values of $13.3(\mathrm{SD} \pm 8.3)$ for the pMMR group $(n=193,65 \%)$ and $9.5(\mathrm{SD} \pm 6)$ for the dMMR group $(n=104,35 \%)(42)$. The higher mean tumor budding count in the dMMR group in our study compared to other studies could be due to the design of the study, by which there might be a higher inclusion of dMMR cases which have recurred or metastasized.

In our study, a significant difference in tumor budding between dMMR and pMMR tumors using the median cutoff of 5 as well as the cut-off of 10 was only found when looking at the dMMR/met+ group compared to the $\mathrm{pMMR} / \mathrm{met}+$ subgroup, in which the $\mathrm{dMMR} / \mathrm{met}+$ group had a higher percentage of high-grade budding.

No benefit of adjuvant chemotherapy according to tumor budding grade was found in this study. However, this was a small retrospective study and was not designed for this purpose. No significant difference in time to recurrence or metastases was seen between the two groups who developed local recurrence or metastases, which is not surprising since the groups were prechosen.

In future studies, it would be of interest to investigate other prognostic factors in addition to tumor budding. One such factor is peritumoral lymphocytic infiltration and its interaction with tumor budding at the invasive front. Several studies have shown that a peritumoral lymphocytic infiltration is an independent prognostic factor $(13,43)$. A quantitative assessment of CD3+ and CD8+ T-lymphocytes resulting in an immunoscore has been used in some studies, where a high a score conferred a better prognosis (44-46). An upcoming goal with our study material is to investigate peritumoral lymphocytic infiltration and its association with the different groups, especially in the high tumor budding cases in the dMMR/met+.

Grading of the tumor inflammatory cell infiltrate has also been done using the Klintrup-Makinen grade $(47,48)$, as well as grading of the tumor stroma percentage (49), and the combination of both resulting in a Glasgow Microenvironment Score (50). An analysis of the latter together with a tumor budding analysis would provide useful prognostic and predictive information.

The assessment of tumor border configuration in primary CRC tumors can also provide additional prognostic information. An infiltrative tumor border is associated with poorer outcome and early disease recurrence compared to a well-demarcated 'pushing' tumor border, which often predicts a better outcome and is mostly seen in dMMR CRCs (51).

As suggested by the study by Zlobec et al. (42), the concept of using a prognostic/predictive score from a combination of markers is a goal to strive for so as to provide patients with the best treatment recommendation. It is important to further explore in larger groups with a more standardized tumor budding assessment method and cut-off whether tumor budding, especially in those with stage II dMMR CRC, is a significant adverse prognostic factor.

\section{Conflicts of Interest}

The Authors report no conflicts of interest in regard to this study. The Authors alone are responsible for the content and writing of the article.

\section{Acknowledgements}

The Authors are grateful for the collaboration with Professor Bengt Glimelius. We thank Yunxia Lu for contributing to the statistical analysis. We also thank Ester Lörinc and Anna Kwiecinska at the Division of Pathology, Karolinska University Hospital for valuable assistance. This study was supported by grants from the Gustaf V Jubilee Fund, the Cancer Society in Stockholm, the Bengt Ihre Foundation of the Swedish Society of Medicine and the Ruth and Rickard Juhlin Foundation. Financial support was also provided through the regional agreement on medical training and clinical research (ALF) between the Stockholm County and Karolinska Institute.

\section{References}

1 Siegel RL, Miller KD and Jemal A: Cancer statistics, 2016. CA Cancer J Clin 66: 7-30, 2016.

2 Labianca R, Nordlinger B, Beretta GD, Mosconi S, Mandala M, Cervantes A, Arnold D and Group EGW: Early colon cancer: ESMO Clinical Practice Guidelines for diagnosis, treatment and follow-up. Ann Oncol 24(Suppl 6): vi64-72, 2013.

3 Edge SB and Compton CC: The American Joint Committee on Cancer: the 7th edition of the AJCC Cancer Staging Manual and the future of TNM. Ann Surg Oncol 17: 1471-1474, 2010.

4 Schmoll HJ, Van Cutsem E, Stein A, Valentini V, Glimelius B, Haustermans K, Nordlinger B, van de Velde CJ, Balmana J, Regula J, Nagtegaal ID, Beets-Tan RG, Arnold D, Ciardiello F, Hoff P, Kerr D, Kohne CH, Labianca R, Price T, Scheithauer W, Sobrero A, Tabernero J, Aderka D, Barroso S, Bodoky G, Douillard JY, El Ghazaly H, Gallardo J, Garin A, Glynne-Jones 
R, Jordan K, Meshcheryakov A, Papamichail D, Pfeiffer P, Souglakos I, Turhal $\mathrm{S}$ and Cervantes A: ESMO Consensus Guidelines for management of patients with colon and rectal cancer. A personalized approach to clinical decision making. Ann Oncol 23: 2479-2516, 2012.

5 Benson AB, 3rd, Schrag D, Somerfield MR, Cohen AM, Figueredo AT, Flynn PJ, Krzyzanowska MK, Maroun J, McAllister P, Van Cutsem E, Brouwers M, Charette M and Haller DG: American Society of Clinical Oncology recommendations on adjuvant chemotherapy for stage II colon cancer. J Clin Oncol 22: 3408-3419, 2004.

6 Marshall JL: Risk assessment in stage II colorectal cancer. Oncology (Williston Park) 24: 9-13, 2010.

7 Popat S, Hubner R and Houlston RS: Systematic review of microsatellite instability and colorectal cancer prognosis. J Clin Oncol 23: 609-618, 2005.

8 Ribic CM, Sargent DJ, Moore MJ, Thibodeau SN, French AJ, Goldberg RM, Hamilton SR, Laurent-Puig P, Gryfe R, Shepherd LE, Tu D, Redston M and Gallinger S: Tumor microsatellite-instability status as a predictor of benefit from fluorouracil-based adjuvant chemotherapy for colon cancer. N Engl J Med 349: 247-257, 2003.

9 Boland CR and Goel A: Microsatellite instability in colorectal cancer. Gastroenterology 138: 2073-2087 e2073, 2010.

10 Lugli A, Karamitopoulou E and Zlobec I: Tumour budding: A promising parameter in colorectal cancer. Br J Cancer 106: 1713-1717, 2012.

11 Mitrovic B, Schaeffer DF, Riddell RH and Kirsch R: Tumor budding in colorectal carcinoma: time to take notice. Mod Pathol 25: 1315-1325, 2012.

12 van Wyk HC, Park J, Roxburgh C, Horgan P, Foulis A and McMillan DC: The role of tumour budding in predicting survival in patients with primary operable colorectal cancer: a systematic review. Cancer Treat Rev 41: 151-159, 2015.

13 Prall F: Tumour budding in colorectal carcinoma. Histopathology 50: 151-162, 2007.

14 Ueno H, Price AB, Wilkinson KH, Jass JR, Mochizuki H and Talbot IC: A new prognostic staging system for rectal cancer. Ann Surg 240: 832-839, 2004.

15 Shinto E, Jass JR, Tsuda H, Sato T, Ueno H, Hase K, Mochizuki $\mathrm{H}$ and Matsubara O: Differential prognostic significance of morphologic invasive markers in colorectal cancer:Tumor budding and cytoplasmic podia. Dis Colon Rectum 49: 1422-1430, 2006.

16 Zlobec I and Lugli A: Epithelial-mesenchymal transition and tumor budding in aggressive colorectal cancer: Tumor budding as oncotarget. Oncotarget 1: 651-661, 2010.

17 Dawson $\mathrm{H}$ and Lugli A: Molecular and pathogenetic aspects of tumor budding in colorectal cancer. Front Med (Lausanne) 2: 11, 2015.

18 Jass JR, Barker M, Fraser L, Walsh MD, Whitehall VL, Gabrielli B, Young J and Leggett BA: APC mutation and tumour budding in colorectal cancer. J Clin Pathol 56: 69-73, 2003.

19 Lugli A, Vlajnic T, Giger O, Karamitopoulou E, Patsouris ES, Peros G, Terracciano LM and Zlobec I: Intratumoral budding as a potential parameter of tumor progression in mismatch repairproficient and mismatch repair-deficient colorectal cancer patients. Hum Pathol 42: 1833-1840, 2011.

20 Petrelli F, Pezzica E, Cabiddu M, Coinu A, Borgonovo K, Ghilardi M, Lonati V, Corti D and Barni S: Tumour budding and survival in stage II colorectal cancer: A systematic review and pooled analysis. J Gastrointest Cancer 46: 212-218, 2015.
21 Lugli A, Kirsch R, Ajioka Y, Bosman F, Cathomas G, Dawson H, El Zimaity H, Flejou JF, Hansen TP, Hartmann A, Kakar S, Langner C, Nagtegaal I, Puppa G, Riddell R, Ristimaki A, Sheahan K, Smyrk T, Sugihara K, Terris B, Ueno H, Vieth M, Zlobec I and Quirke P: Recommendations for reporting tumor budding in colorectal cancer based on the International Tumor Budding Consensus Conference (ITBCC) 2016. Mod Pathol 30: 1299-1311, 2017.

22 Prall F, Nizze $\mathrm{H}$ and Barten M: Tumour budding as prognostic factor in stage I/II colorectal carcinoma. Histopathology 47: 17$24,2005$.

23 Ohtsuki K, Koyama F, Tamura T, Enomoto Y, Fujii H, Mukogawa T, Nakagawa $T$, Uchimoto $K$, Nakamura $S$, Nonomura A and Nakajima Y: Prognostic value of immunohistochemical analysis of tumor budding in colorectal carcinoma. Anticancer Res 28: 1831-1836, 2008.

24 Koelzer VH, Zlobec I, Berger MD, Cathomas G, Dawson H, Dirschmid K, Hadrich M, Inderbitzin D, Offner F, Puppa G, Seelentag W, Schnuriger B, Tornillo L and Lugli A: Tumor budding in colorectal cancer revisited: results of a multicenter interobserver study. Virchows Arch 466: 485-493, 2015.

25 Rieger G, Koelzer VH, Dawson HE, Berger MD, Hadrich M, Inderbitzin D, Lugli A and Zlobec I: Comprehensive assessment of tumour budding by cytokeratin staining in colorectal cancer. Histopathology 70: 1044-1051, 2017.

26 Puppa G, Senore C, Sheahan K, Vieth M, Lugli A, Zlobec I, Pecori S, Wang LM, Langner C, Mitomi H, Nakamura T, Watanabe M, Ueno H, Chasle J, Conley SA, Herlin P, Lauwers GY and Risio M: Diagnostic reproducibility of tumour budding in colorectal cancer: A multicentre, multinational study using virtual microscopy. Histopathology 61: 562-575, 2012.

27 Rogers AC, Winter DC, Heeney A, Gibbons D, Lugli A, Puppa $\mathrm{G}$ and Sheahan K: Systematic review and meta-analysis of the impact of tumour budding in colorectal cancer. Br J Cancer 115: 831-840, 2016.

28 Markl B and Arnholdt HM: Prognostic significance of tumor budding in gastrointestinal tumors. Expert Rev Anticancer Ther 11: 1521-1533, 2011.

29 Glimelius B, Dahl O, Cedermark B, Jakobsen A, Bentzen SM, Starkhammar H, Gronberg H, Hultborn R, Albertsson M, Pahlman L and Tveit KM: Adjuvant chemotherapy in colorectal cancer: a joint analysis of randomised trials by the Nordic Gastrointestinal Tumour Adjuvant Therapy Group. Acta Oncol 44: 904-912, 2005.

30 Ohrling K, Edler D, Hallstrom M and Ragnhammar P: Mismatch repair protein expression is an independent prognostic factor in sporadic colorectal cancer. Acta Oncol 49: 797-804, 2010.

31 Ueno H, Murphy J, Jass JR, Mochizuki H and Talbot IC: Tumour 'budding' as an index to estimate the potential of aggressiveness in rectal cancer. Histopathology 40: 127-132, 2002.

32 Graham RP, Vierkant RA, Tillmans LS, Wang AH, Laird PW, Weisenberger DJ, Lynch CF, French AJ, Slager SL, Raissian Y, Garcia JJ, Kerr SE, Lee HE, Thibodeau SN, Cerhan JR, Limburg PJ and Smyrk TC: Tumor budding in colorectal carcinoma: confirmation of prognostic significance and histologic cutoff in a population-based cohort. Am J Surg Pathol 39: 1340-1346, 2015.

33 Karamitopoulou E, Zlobec I, Kolzer V, Kondi-Pafiti A, Patsouris ES, Gennatas K and Lugli A: Proposal for a 10-high-powerfields scoring method for the assessment of tumor budding in colorectal cancer. Mod Pathol 26: 295-301, 2013. 
34 Lindor NM, Burgart LJ, Leontovich O, Goldberg RM, Cunningham JM, Sargent DJ, Walsh-Vockley C, Petersen GM, Walsh MD, Leggett BA, Young JP, Barker MA, Jass JR, Hopper J, Gallinger S, Bapat B, Redston $\mathrm{M}$ and Thibodeau SN: Immunohistochemistry versus microsatellite instability testing in phenotyping colorectal tumors. J Clin Oncol 20: 1043-1048, 2002.

35 Kevans D, Wang LM, Sheahan K, Hyland J, O'Donoghue D, Mulcahy $\mathrm{H}$ and O'Sullivan J: Epithelial-mesenchymal transition (EMT) protein expression in a cohort of stage II colorectal cancer patients with characterized tumor budding and mismatch repair protein status. Int J Surg Pathol 19: 751-760, 2011.

36 van Wyk HC, Park JH, Edwards J, Horgan PG, McMillan DC and Going JJ: The relationship between tumour budding, the tumour microenvironment and survival in patients with primary operable colorectal cancer. Br J Cancer 115: 156-163, 2016.

37 Shinto E, Baker K, Tsuda H, Mochizuki H, Ueno H, Matsubara O, Foulkes WD and Jass JR: Tumor buds show reduced expression of laminin-5 gamma 2 chain in DNA mismatch repair-deficient colorectal cancer. Dis Colon Rectum 49: 1193 1202, 2006.

38 Zlobec I: Novel biomarkers for the prediction of metastasis in colorectal cancer. Expert Opin Med Diagn 7: 137-146, 2013.

39 Park KJ, Choi HJ, Roh MS, Kwon HC and Kim C: Intensity of tumor budding and its prognostic implications in invasive colon carcinoma. Dis Colon Rectum 48: 1597-1602, 2005.

40 Wang LM, Kevans D, Mulcahy H, O'Sullivan J, Fennelly D, Hyland J, O'Donoghue D and Sheahan K: Tumor budding is a strong and reproducible prognostic marker in T3N0 colorectal cancer. Am J Surg Pathol 33: 134-141, 2009.

41 Nakamura T, Mitomi H, Kikuchi S, Ohtani Y and Sato K: Evaluation of the usefulness of tumor budding on the prediction of metastasis to the lung and liver after curative excision of colorectal cancer. Hepatogastroenterology 52: 1432-1435, 2005.

42 Zlobec I, Minoo P, Terracciano L, Baker K and Lugli A: Characterization of the immunological microenvironment of tumour buds and its impact on prognosis in mismatch repairproficient and -deficient colorectal cancers. Histopathology 59: 482-495, 2011.

43 Lugli A, Karamitopoulou E, Panayiotides I, Karakitsos P, Rallis G, Peros G, Iezzi G, Spagnoli G, Bihl M, Terracciano L and Zlobec I: CD8+ lymphocytes/tumour-budding index: an independent prognostic factor representing a 'pro-/anti-tumour' approach to tumour host interaction in colorectal cancer. $\mathrm{Br} \mathrm{J}$ Cancer 101: 1382-1392, 2009.

44 Park JH, McMillan DC, Edwards J, Horgan PG and Roxburgh CS: Comparison of the prognostic value of measures of the tumor inflammatory cell infiltrate and tumor-associated stroma in patients with primary operable colorectal cancer. Oncoimmunology 5: e1098801, 2016
45 Galon J, Mlecnik B, Bindea G, Angell HK, Berger A, Lagorce C, Lugli A, Zlobec I, Hartmann A, Bifulco C, Nagtegaal ID, Palmqvist R, Masucci GV, Botti G, Tatangelo F, Delrio P, Maio M, Laghi L, Grizzi F, Asslaber M, D'Arrigo C, VidalVanaclocha F, Zavadova E, Chouchane L, Ohashi PS, HafeziBakhtiari S, Wouters BG, Roehrl M, Nguyen L, Kawakami Y, Hazama S, Okuno K, Ogino S, Gibbs P, Waring P, Sato N, Torigoe T, Itoh K, Patel PS, Shukla SN, Wang Y, Kopetz S, Sinicrope FA, Scripcariu V, Ascierto PA, Marincola FM, Fox BA and Pages F: Towards the introduction of the 'Immunoscore' in the classification of malignant tumours. J Pathol 232: 199-209, 2014.

46 Mlecnik B, Bindea G, Angell HK, Maby P, Angelova M, Tougeron D, Church SE, Lafontaine L, Fischer M, Fredriksen T, Sasso M, Bilocq AM, Kirilovsky A, Obenauf AC, Hamieh M, Berger A, Bruneval P, Tuech JJ, Sabourin JC, Le Pessot F, Mauillon J, Rafii A, Laurent-Puig P, Speicher MR, Trajanoski Z, Michel P, Sesboue R, Frebourg T, Pages F, Valge-Archer V, Latouche JB and Galon J: Integrative analyses of colorectal cancer show immunoscore is a stronger predictor of patient survival than microsatellite instability. Immunity 44: 698-711, 2016.

47 Klintrup K, Makinen JM, Kauppila S, Vare PO, Melkko J, Tuominen H, Tuppurainen K, Makela J, Karttunen TJ and Makinen MJ: Inflammation and prognosis in colorectal cancer. Eur J Cancer 41: 2645-2654, 2005.

48 Roxburgh CS, Salmond JM, Horgan PG, Oien KA and McMillan DC: Tumour inflammatory infiltrate predicts survival following curative resection for node-negative colorectal cancer. Eur $\mathrm{J}$ Cancer 45: 2138-2145, 2009.

49 Park JH, Richards CH, McMillan DC, Horgan PG and Roxburgh CS: The relationship between tumour stroma percentage, the tumour microenvironment and survival in patients with primary operable colorectal cancer. Ann Oncol 25: 644-651, 2014.

50 McMillan DC: The systemic inflammation-based Glasgow Prognostic Score: a decade of experience in patients with cancer. Cancer Treat Rev 39: 534-540, 2013.

51 Koelzer VH and Lugli A: The tumor border configuration of colorectal cancer as a histomorphological prognostic indicator. Front Oncol 4: 29, 2014.
Received May 28, 2018

Revised June 22, 2018

Accepted July 3, 2018 